\title{
Prognostic value of osteopontin expression in breast cancer: A meta-analysis
}

\author{
YING-YI XU $^{1 *}$, YA-YA ZHANG $^{1 *}$, WEI-FENG LU ${ }^{2}$, YAN-JUN MI $^{1}$ and YU-QIANG CHEN ${ }^{1}$ \\ ${ }^{1}$ Department of Oncology, Chenggong Hospital Affiliated to Xiamen University, No. 174 Hospital of People's Liberation Army; \\ ${ }^{2}$ Department of Vascular Surgery, Zhongshan Hospital, Xiamen 361003, P.R. China
}

Received September 22, 2014; Accepted November 14, 2014

DOI: $10.3892 / \operatorname{mco} .2014 .480$

\begin{abstract}
Osteopontin (OPN) has been implicated in tumor development and progression over the last few years. However, the prognostic value of OPN overexpression in patients with breast cancer remains controversial. We performed a meta-analysis to investigate the association of OPN expression in the tumor with the clinicopathological characteristics and survival of breast cancer patients. A total of 8 studies met the inclusion criteria and were entered in the meta-analysis. The data analysis demonstrated that OPN expression was positively associated with lymph node metastasis [pooled odds ratio $=2.026,95 \%$ confidence interval $(\mathrm{CI}): 1.199-3.425$, $\mathrm{P}=0.008$, random-effects model]. We also found that OPN expression was positively associated with overall survival [hazard ratio $(\mathrm{HR})=3.69,95 \% \mathrm{CI}: 1.45-9.42, \mathrm{P}=0.000$, random-effects model) and disease-free survival (pooled $\mathrm{HR}=2.40,95 \% \mathrm{CI}: 1.27-4.55, \mathrm{P}=0.007$, fixed-effects model). Based on the results of this study, we concluded that OPN overexpression in the tumor is a candidate positive prognostic biomarker for breast cancer patients.
\end{abstract}

\section{Introduction}

Breast cancer is the most commonly diagnosed cancer in women. Up to 2013, breast cancer reportedly accounted for $29 \%$ of all new cancer cases and $14 \%$ of all cancer-related deaths among women worldwide (1). Breast cancer-related mortality is closely associated with the development of metastatic potential of the primary tumor. Despite the significant efforts to reduce breast cancer metastasis and mortality, its prognosis remains poor. Therefore, we must identify novel biomarkers to improve the prognosis of breast cancer. It was previously

Correspondence to: Dr Yu-Qiang Chen, Department of Oncology, Chenggong Hospital Affiliated to Xiamen University, No. 174 Hospital of People's Liberation Army, 94-96 Wenyuan Road, Xiamen 361003, P.R. China

E-mail: chenyq707@163.com

*Contributed equally

Key words: breast cancer, osteopontin, prognosis, meta-analysis demonstrated that the expression of osteopontin (OPN) may reflect breast cancer progression and metastasis (2).

OPN is a secreted glycophosphoprotein that has been implicated in physiological as well as pathological processes (3). Over the last few years, a number of studies have reported that aberrant OPN expression is closely associated with tumorigenesis and metastasis in several tumors, including breast cancer (4-6). In addition, several studies demonstrated that OPN overexpression was associated with poor prognosis of patients with breast cancer (7-11). OPN may also be used in conjunction with other markers of known prognostic and predictive value that are commonly evaluated in the primary tumor, such as estrogen receptors (ER), progesterone receptors (PR) and HER2 (12,13). However, the emerging data regarding the ability of OPN to predict disease progression, overall survival (OS) and disease-free survival (DFS) in patients with breast cancer have been inconsistent. To address this issue, we conducted a meta-analysis aimed at estimating the value of OPN as a prognostic marker for breast cancer and to confirm the association between OPN and several clinicopathological characteristics of breast cancer.

\section{Materials and methods}

Study selection. A search was conducted through the PubMed electronic database to identify studies investigating the association of clinicopathological parameters and prognosis with OPN expression in breast cancer up to February 14, 2014, for inclusion in the present meta-analysis. The search terms were 'breast cancer' and 'OPN'. The published studies that were included in this meta-analysis were required to meet the following criteria: i) the type of tumor investigated was breast cancer; ii) the studies assessed the association between OPN expression and clinicopathological characteristics and/or survival and only full peer-reviewed papers had been published as full texts; and iii) the protein expression was evaluated in tumor tissues by immunohistochemistry (IHC). There were no limitations regarding the language or the patient numbers.

Data extraction. Two investigators independently reviewed all potentially relevant studies to minimize bias and improve reliability. The following data were extracted from the eligible studies: name of first author, name of journal, year of publication, sample size, test method, cut-off value, age, HER2, 


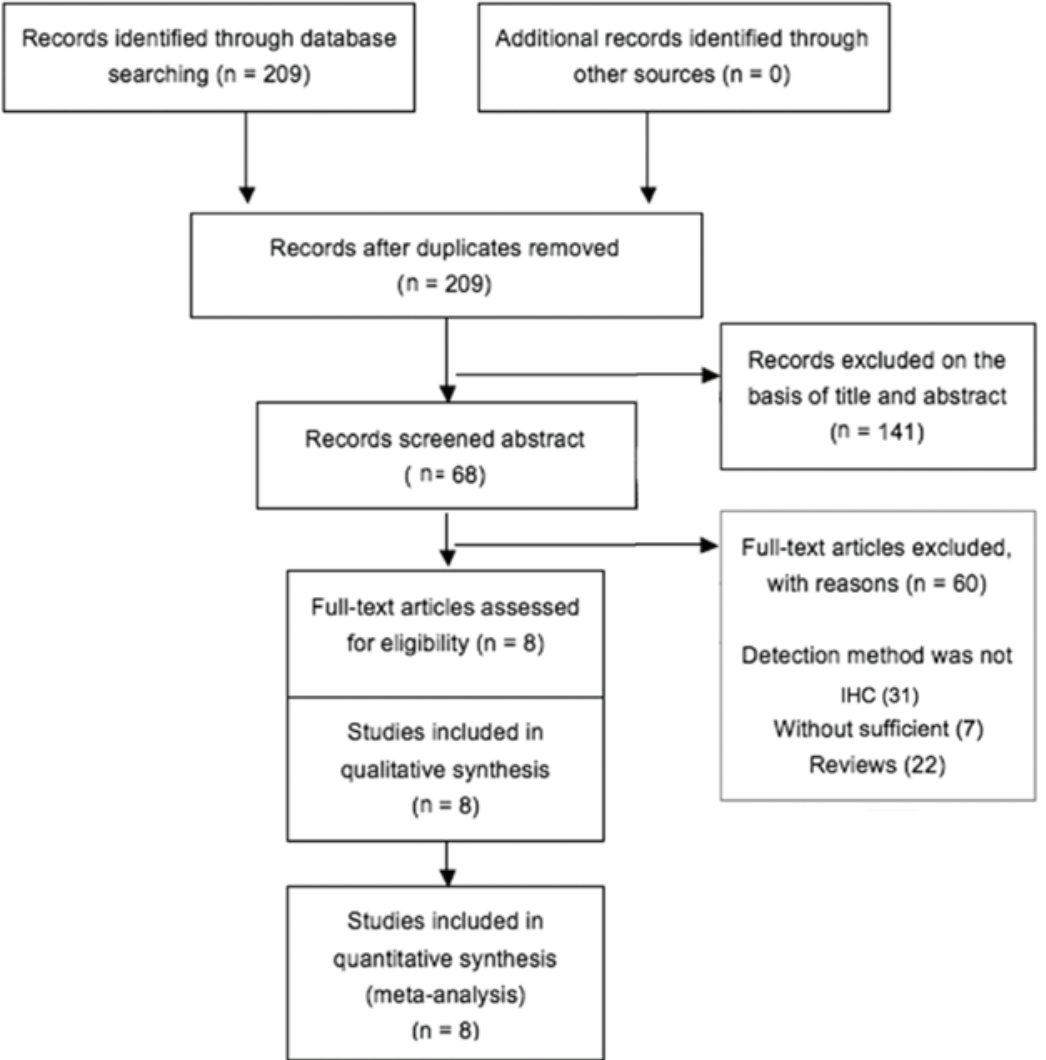

Figure 1. Flow chart of the selection process of studies for inclusion in this meta-analysis.

PR, ER, p53, menopausal status, differentiation, lymph node metastasis, stage and OPN expression-related survival. When the prognosis was plotted as a Kaplan-Meier curve, the GetData Graph Digitizer 2.24 software (http://getdata-graph-digitizer. com) and HR digitizer Engauge 4.0 software (http://engaugedigitizer.software.informer.com/) were used to digitize and extract the data. In brief, the Kaplan-Meier curve was saved as a graph and opened in the GetData Graph Digitizer 2.24 and Engauge Digitizer 4.0 software, the scale was set (coordinate system) and, finally, the points of the Kaplan-Meier curve were manually digitized.

Quality assessment. The methodological quality of each included study was assessed on basis of the Newcastle-Ottawa scale (NOS) (14) by two independent reviewers. A star or point system of NOS has been developed for the evaluation. The scores are shown in Table I. The publications included in our meta-analysis, with scores of $\geq 5$, were rated as high-quality.

Statistical analysis. All the statistical analyses were performed using Stata 12.0 software for Windows (Stata Corporation, College Station, TX, USA). The pooled estimates of odds ratios (ORs) with their 95\% confidence intervals (CIs) were applied to assess the association between OPN expression and the clinical parameters of breast cancer, including age, HER2, PR, ER, p53, menopausal status, differentiation, lymph node metastasis and stage. The pooled estimates of hazard ratios (HRs) with their 95\% CIs were applied to estimate the association between OPN expression and the survival outcome of breast cancer. The statistical heterogeneity within studies was detected with the Chi-squared based Q-test $(\mathrm{P}>0.10)$ and $\mathrm{I}^{2}$. When $\mathrm{I}^{2}<50 \%$, there was no heterogeneity and the fixed-effects model was used. In the opposite case, the random-effects model was used. The assessment of publication bias was performed by Egger's and Begg's tests and the potential publication bias was deemed significant at $\mathrm{P}<0.05$.

\section{Results}

Description of studies. Following a combined search in PubMed using the terms 'breast cancer' and 'OPN' 209 potentially relevant citations were retrieved. After excluding animal experiments, non-breast cancer-related studies, non-original articles, or studies lacking data on the association of OPN with clinicopathological characteristics and/or OS, only 8 studies met the inclusion criteria for the present analysis (Fig. 1). Two of these publications lacked information on survival and follow-up and could not be included in the survival analysis. The sample size ranged between 100 and 333 patients. The expression of OPN was detected by IHC in all the studies. Detailed information on the parameters of the included publications is provided in Table I.

Correlation of OPN expression with clinicopathological characteristics. The main results of the meta-analysis are summarized in Table II. In certain studies, presented data on clinicopathological characteristics could not be extracted and when parameters were provided by $>3$ publications, a meta-analysis was performed. There was no correlation between OPN expression and age (pooled $\mathrm{OR}=1.132$, 95\% CI: 0.737-1.737, 

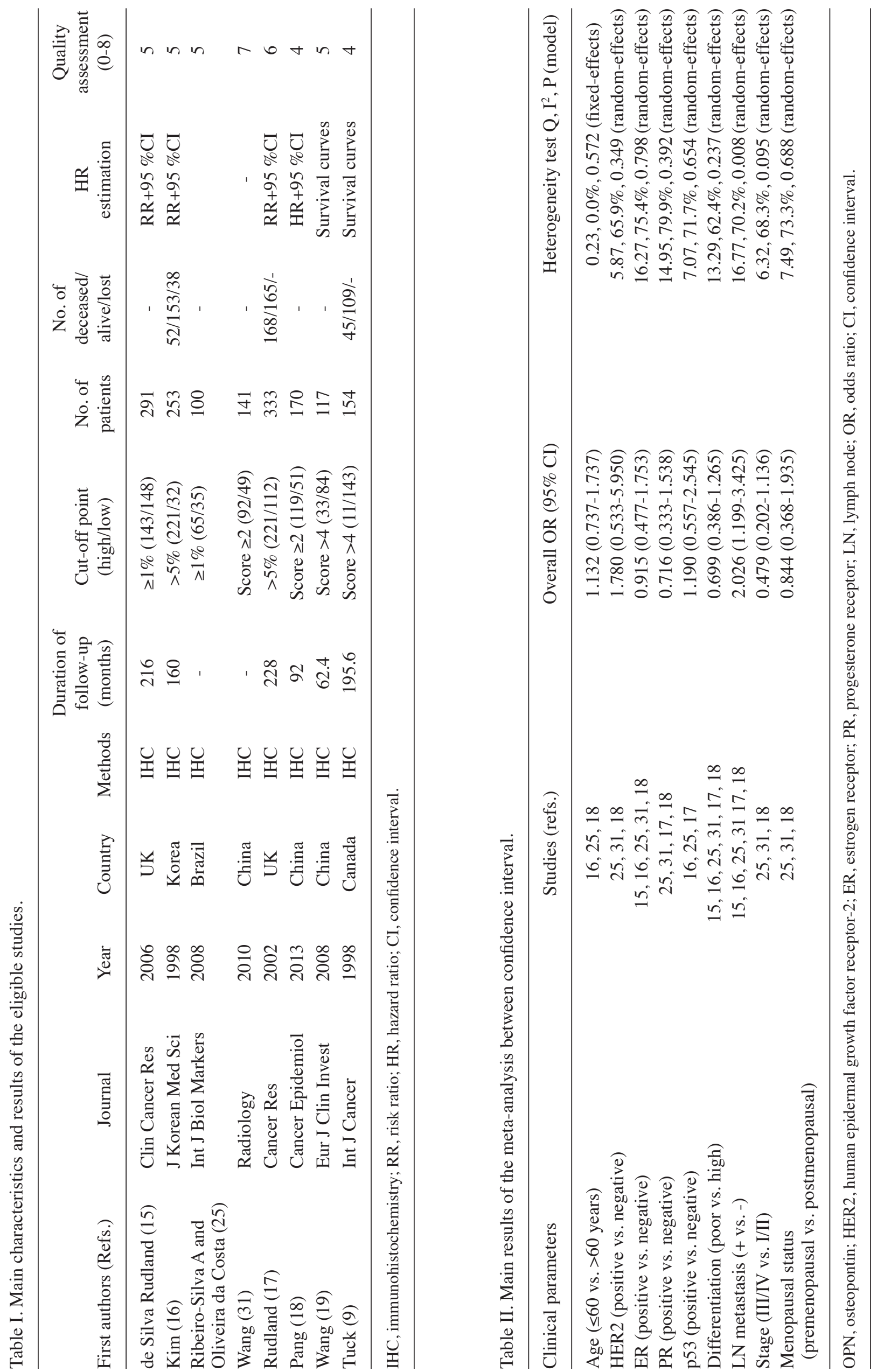


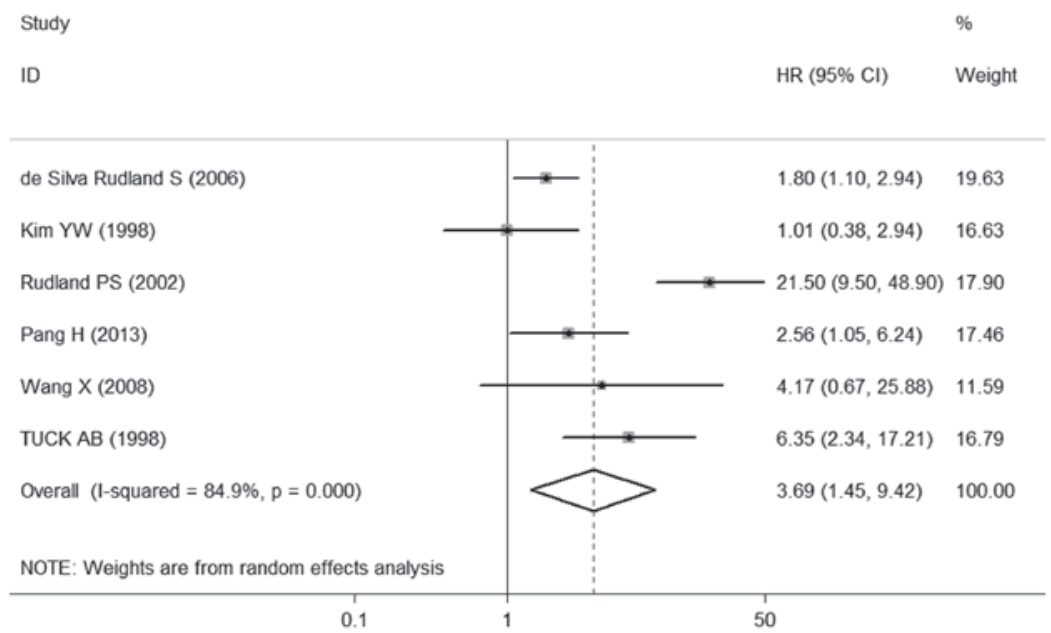

Figure 2. Forest plot demonstrating the association of osteopontin expression with overall survival in breast cancer. HR, hazard ratio; CI, confidence interval.

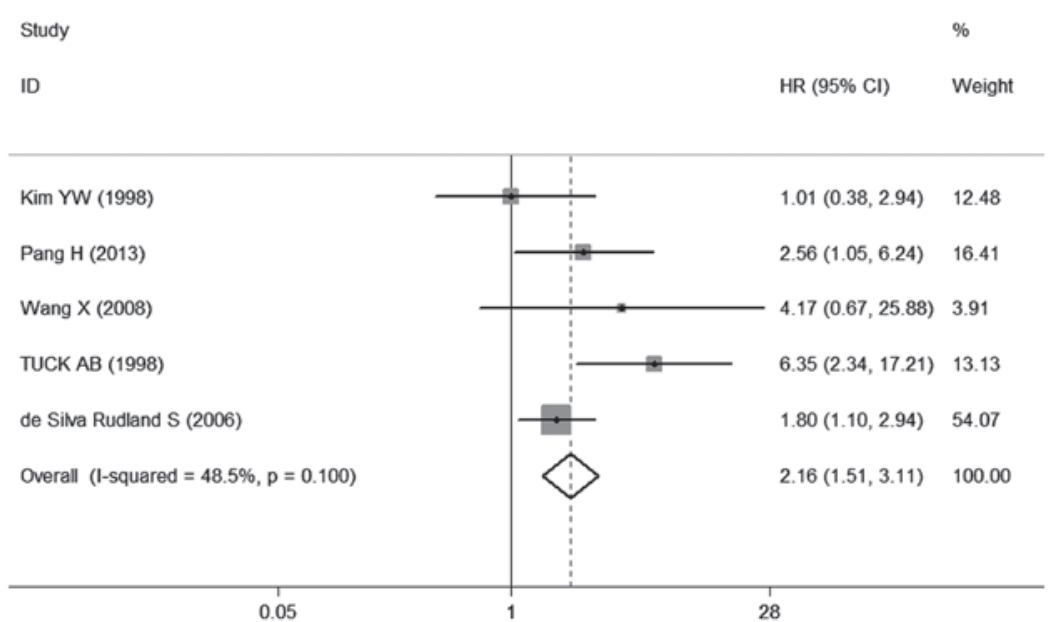

Figure 3. Forest plot on the association between osteopontin expression and overall survival in breast cancer by sensitivity analysis. HR, hazard ratio; CI, confidence interval.

$\mathrm{P}=0.572$ ), HER2 (pooled $\mathrm{OR}=1.780,95 \% \mathrm{CI}: 0.533-5.950$, $\mathrm{P}=0.349)$, PR (pooled OR=0.716, 95\% CI: 0.333-1.538, $\mathrm{P}=0.392), \mathrm{ER}$ (pooled $\mathrm{OR}=0.915,95 \% \mathrm{CI}: 0.477-1.753, \mathrm{P}=0.798$ ), p53 (pooled OR=1.190, 95\% CI: 0.557-2.545, $\mathrm{P}=0.654$ ), menopausal status (pooled $\mathrm{OR}=0.844,95 \% \mathrm{CI}$ : 0.368-1.935, $\mathrm{P}=0.688$ ), tumor differentiation (pooled $\mathrm{OR}=0.699$, 95\% CI: $0.386-1.265, \mathrm{P}=0.237$ ) and stage (pooled $\mathrm{OR}=0.479$, 95\% CI: 0.202-1.136, $\mathrm{P}=0.095)$. However, OPN expression was positively correlated with lymph node metastasis compared to the absence of lymph node metastasis. (pooled OR=2.026, 95\% CI: 1.199-3.425, $\mathrm{P}=0.008$ ).

Effect of OPN expression on OS of breast cancer. The different results were extracted from previous studies on the effect of OPN expression on OS. A total of 6 studies $(9,15-19)$ provided data on OS. All 6 studies investigating OS were pooled into the meta-analysis. The results demonstrated that OPN overexpression was correlated with poor OS $(\mathrm{HR}=3.69$, 95\% CI: $1.45-9.42, \mathrm{P}=0.000$ ) (Fig. 2), with heterogeneity in the data $\left(\chi^{2}=33.04, \mathrm{I}^{2}=84.9 \%, \mathrm{P}=0.000\right)$. In order to test the heterogeneity in OS, we performed sensitivity analyses to assess the stability of the results. Our results suggested that the hetero- geneity for OS was significantly decreased by exclusion of the study of Rudland et al (17) $\left(\chi^{2}=7.77, \mathrm{I}^{2}=48.5 \%, \mathrm{P}=0.100\right)$, with a combined OR of 2.16 (95\% CI: 1.51-3.11, $\mathrm{P}=0.000$ ) (Fig. 3), demonstrating that the sensitivity is low and the result is more robust and credible.

Effect of OPN expression on DFS of breast cancer. The different results were extracted from previous studies on the effect of OPN expression on DFS. A total of 3 studies $(9,18,19)$ provided information on DFS. The combined data of the 3 studies indicated that patients with OPN overexpression exhibited a shorter DFS (pooled HR=2.40, 95\% CI: 1.27-4.56, $\mathrm{P}=0.007$ ) (Fig. 4), without any heterogeneity in the data $\left(\chi^{2}=0.08, \mathrm{I}^{2}=0.0 \%\right.$, $\mathrm{P}=0.959)$, suggesting that the sensitivity is low and the result is more robust and credible. These studies indicated that OPN overexpression is associated with the prognosis of breast cancer.

Publication bias. Begg's funnel plot with pseudo 95\% confidence limits and Egger's test were applied to estimate the publication bias of the included literature (Figs. 5 and 6). Begg's and Egger's tests did not reveal any evidence of obvious asymmetry in the overall meta-analysis of all the studies. 


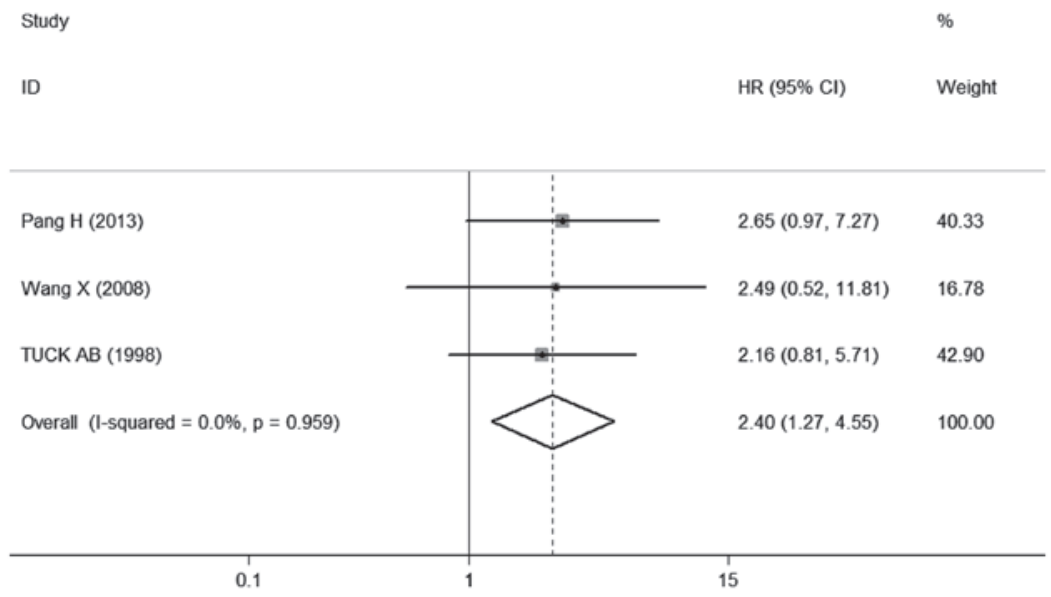

Figure 4. Forest plot demonstrating the association of osteopontin expression with disease-free survival in breast cancer. HR, hazard ratio; CI, confidence interval.

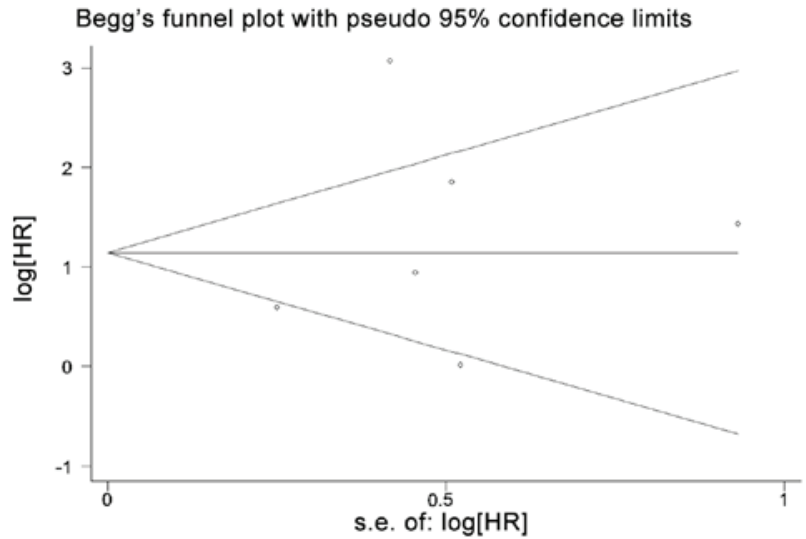

Figure 5. Begg's funnel plot estimating the publication bias of the included studies. HR, hazard ratio.

\section{Discussion}

Meta-analyses based on individual data are currently considered the golden standard for systematic reviews (20). Meta-analysis has been successfully applied in the evaluation of prognostic indicators in patients with malignant diseases (21-24). To the best of our knowledge, this meta-analysis is the first to systematically review the association of OPN with clinicopathological parameters and prognostic factors in breast cancer.

In our study, a combined analysis of all the included articles that compared breast cancer patients according to the tumor expression of OPN, revealed a poor prognostic outcome in patients expressing high levels of OPN. OPN is a prognostic factor in breast cancer; although OPN overexpression was reportedly associated with lower tumor grade $(17,18)$ and lower tumor stage (18), PR status $(18,25)$, ER status (25) and p53 status (17), our results indicated that the positive expression of OPN was not correlated with these clinicopathological parameters. The results indicated that OPN expression is significantly associated with lymph node metastasis, OS and DFS. Tumor invasion and metastasis have been frequently correlated with poor prognosis of breast cancer $(26,27)$, as described above. Previous studies have reported high OPN levels in the blood and primary tumors of breast cancer patients and, in certain cases, this was correlated with a poor prognosis $(6,9,10,17)$.

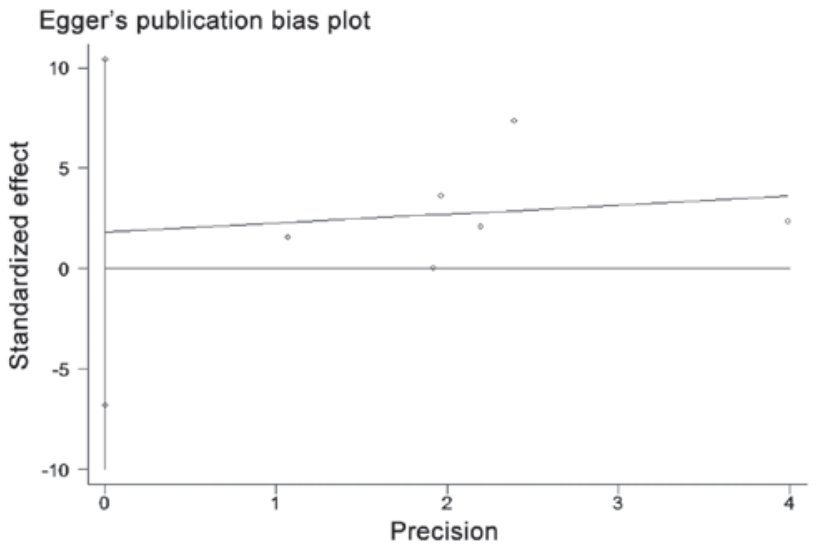

Figure 6. Egger's funnel plot estimating the publication bias of the included studies. HR, hazard ratio.

A highly metastatic human breast cancer cell line was shown to express significantly higher levels of OPN compared to a cell line of low metastatic potential (28). Dai et al (29) used a humanized OPN-specific antibody to effectively prevent breast cancer cell invasion in vitro and inhibit pulmonary metastasis of breast cancer in nude mice. OPN may act as a diagnostic marker as well as a potential therapeutic target for breast cancer metastasis. Tuck et al (30) demonstrated that OPN was crucial for the metastasis of breast cancer, which may be regulated through activation of the protein kinase $\mathrm{C}$, phospholipase $\mathrm{C}$ and phosphoinositide 3-kinase pathways. Consequently, OPN may be a marker for poor prognosis and lymph node metastasis in breast cancer.

There were several important limitations to this meta-analysis. First, we searched studies only from one database and the number of included studies was relatively small. Second, 4 of the 8 studies were from Asia ( 3 from China and 1 from Korea), 3 were from Europe and 1 was from Brazil. Distinct differences among different locations may result in publication bias. Third, the cut-off values for determining the positive or negative expression of OPN were different among the included studies, which may contribute to heterogeneity. New studies with the same cut-off values must be included in a combined analysis for further evaluation. Finally, there are only a few studies specifically investigating OPN and the prognosis of breast cancer. 
In summary, in this meta-analysis, we demonstrated that OPN expression was not correlated with clinicopathological parameters, apart from lymph node metastasis. Therefore, we conclude that OPN overexpression is associated with poor OS and DFS and may serve as a potential molecular target for the clinical management of breast cancer.

\section{Acknowledgements}

This study was supported by the National Natural Scientific Foundation of China (grant no. 81100225).

\section{References}

1. Siegel R, Naishadham D and Jemal A: Cancer statistics, 2013. CA Cancer J Clin 63: 11-30, 2013.

2. Furger KA, Menon RK, Tuck AB, Bramwell VH and Chambers AF: The functional and clinical roles of osteopontin in cancer and metastasis. Curr Mol Med 1: 621-632, 2001.

3. Denhardt DT and Guo X: Osteopontin: a protein with diverse functions. FASEB J 7: 1475-1482, 1993.

4. Brown LF, Papadopoulos-Sergiou A, Berse B, et al: Osteopontin expression and distribution in human carcinomas. Am J Pathol 145: 610-623, 1994.

5. Agrawal D, Chen T, Irby R, et al: Osteopontin identified as lead marker of colon cancer progression, using pooled sample expression profiling. J Natl Cancer Inst 94: 513-521, 2002.

6. Coppola D, Szabo M, Boulware D, et al: Correlation of osteopontin protein expression and pathological stage across a wide variety of tumor histologies. Clin Cancer Res 10: 184-190, 2004

7. Tuck AB and Chambers AF: The role of osteopontin in breast cancer: clinical and experimental studies. J Mammary Gland Biol Neoplasia 6: 419-429, 2001.

8. Tuck AB, Chambers AF and Allan AL: Osteopontin overexpression in breast cancer: knowledge gained and possible implications for clinical management. J Cell Biochem 102: 859-868, 2007.

9. Tuck AB, O'Malley FP, Singhal H, et al: Osteopontin expression in a group of lymph node negative breast cancer patients. Int $\mathrm{J}$ Cancer 79: 502-508, 1998.

10. Singhal H, Bautista DS, Tonkin KS, et al: Elevated plasma osteopontin in metastatic breast cancer associated with increased tumor burden and decreased survival. Clin Cancer Res 3: 605-611, 1997.

11. Bellahcène A and Castronovo V: Increased expression of osteonectin and osteopontin, two bone matrix proteins, in human breast cancer. Am J Pathol 146: 95-100, 1995.

12. Bramwell VH, Doig GS, Tuck AB, et al: Serial plasma osteopontin levels have prognostic value in metastatic breast cancer. Clin Cancer Res 12: 3337-3343, 2006.

13. Chang J, Clark GM, Allred DC, Mohsin S, Chamness G and Elledge RM: Survival of patients with metastatic breast carcinoma: importance of prognostic markers of the primary tumor. Cancer 97: 545-553, 2003.

14. Stang A: Critical evaluation of the Newcastle-Ottawa scale for the assessment of the quality of nonrandomized studies in meta-analyses. Eur J Epidemiol 25: 603-605, 2010.
15. de Silva Rudland S, Martin L, Roshanlall C, et al: Association of S100A4 and osteopontin with specific prognostic factors and survival of patients with minimally invasive breast cancer. Clin Cancer Res 12: 1192-1200, 2006.

16. Kim YW, Park YK, Lee J, Ko SW and Yang MH: Expression of osteopontin and osteonectin in breast cancer. J Korean Med Sci 13: 652-657, 1998.

17. Rudland PS, Platt-Higgins A, El-Tanani M, et al: Prognostic significance of the metastasis-associated protein osteopontin in human breast cancer. Cancer Res 62: 3417-3427, 2002.

18. Pang H, Lu H, Song H, et al: Prognostic values of osteopontin-c, E-cadherin and beta-catenin in breast cancer. Cancer Epidemiol 37: 985-992, 2013

19. Wang X, Chao L, Ma G, et al: Increased expression of osteopontin in patients with triple-negative breast cancer. Eur J Clin Invest 38: 438-446, 2008.

20. Stewart LA and Parmar MK: Meta-analysis of the literature or of individual patient data: is there a difference? Lancet 341: 418-422, 1993.

21. Liu LY, Wang M, Ma ZB, et al: The role of adiponectin in breast cancer: a meta-analysis. PLoS One 8: e73183, 2013.

22. Lum DW, Perel P, Hingorani AD and Holmes MV: CYP2D6 genotype and tamoxifen response for breast cancer: a systematic review and meta-analysis. PLoS One 8: e76648, 2013.

23. Qu Q, Mao Y, Fei XC and Shen KW: The impact of androgen receptor expression on breast cancer survival: a retrospective study and meta-analysis. PLoS One 8: e82650, 2013.

24. Zhang Z, Ni C, Chen W, et al: Expression of CXCR4 and breast cancer prognosis: a systematic review and meta-analysis. BMC Cancer 14: 49, 2014.

25. Ribeiro-Silva A and Oliveira da Costa JP: Osteopontin expression according to molecular profile of invasive breast cancer: a clinicopathological and immunohistochemical study. Int J Biol Markers 23: 154-160, 2008

26. Allan AL, George R, Vantyghem SA, et al: Role of the integrin-binding protein osteopontin in lymphatic metastasis of breast cancer. Am J Pathol 169: 233-246, 2006.

27. Chambers AF, Groom AC and MacDonald IC: Dissemination and growth of cancer cells in metastatic sites. Nat Rev Cancer 2: 563-572, 2002.

28. Tuck AB, Arsenault DM, O'Malley FP, et al: Osteopontin induces increased invasiveness and plasminogen activator expression of human mammary epithelial cells. Oncogene 18: 4237-4246, 1999.

29. Dai J, Li B, Shi J, et al: A humanized anti-osteopontin antibody inhibits breast cancer growth and metastasis in vivo. Cancer Immunol Immunother 59: 355-366, 2010.

30. Tuck AB, Hota C, Wilson SM and Chambers AF: Osteopontin-induced migration of human mammary epithelial cells involves activation of EGF receptor and multiple signal transduction pathways. Oncogene 22: 1198-1205, 2003.

31. Wang X, Chao L, Ma G, et al: Primary breast carcinoma: association of mammographic calcifications with osteopontin expression.. Radiology 254: 69-78, 2010. 